\title{
Fachkräftemangel im Handwerk? - eine Spurensuche
}

Mit Blick auf die kleinbetrieblich geprägte Handwerkswirtschaft besteht in der öffentlichen Diskussion die Sorge vor einem ausgeprägten „Fachkräftemangel“. Es fehlt in diesem Zusammenhang jedoch an wissenschaftlich fundierten Einschätzungen, die sich mit diesem Sachverhalt auseinandersetzen. Indem aus einer Vergleichsperspektive verschiedene empirische Befunde gesammelt und hinsichtlich ihrer Implikationen für die handwerkliche Nachwuchs- und Fachkräftesicherung bewertet werden, möchte der vorliegende Beitrag zur Beantwortung der Frage beitragen, ob eine bedarfsdeckende Fachkräfteversorgung aktuell und in der näheren Zukunft insbesondere für Handwerksbetriebe eine Herausforderung darstellt. ${ }^{1}$

JÖRG THOMÄ

\section{Einführung}

In der aktuellen öffentlichen Diskussion zur Lage am deutschen Arbeitsmarkt überwiegt die Wahrnehmung, dass ein bereits bestehender, allemal sich künftig entwickelnder „Fachkräftemangel“ eines der gravierenden arbeitsmarktpolitischen Probleme sei. Diese Einschätzung fußt einerseits auf den gestiegenen Qualifikationsanforderungen an Beschäftigte, andererseits auf der Verknappung bzw. Alterung des verfügbaren Arbeitskräfteangebots im Zuge des demografischen Wandels. Die These von einer strukturellen Fachkräftelücke in Deutschland - im Sinne eines dauerhaften Überschusses der Arbeitsnachfrage über das Arbeitsangebot - lässt sich jedoch derzeit mit Zahlen kaum belegen. Und auch künftig ist es kaum realistisch, dass ein solches Negativszenario in dem vielfach prognostizierten Umfang eintritt (IAB-Autorengruppe 2012). Gleichwohl kommt es bereits heute je nach Beruf, nachgefragter Qualifikation oder Region mitunter zu spürbaren Engpässen bei der Rekrutierung von qualifiziertem Personal. Durch den demografisch bedingten Rückgang des künftigen Erwerbspersonenpotenzials und vor allem im Falle eines Ausbleibens von Gegenmaßnahmen, die auf eine bessere Mobilisierung von inländischen Potenzialen und eine stärkere Zuwanderung von ausländischen Fachkräften zielen, dürfte diese Situation zukünftig noch an Brisanz gewinnen.
In diesem Zusammenhang wird gerade mit Blick auf kleine und mittlere Unternehmen (KMU) eine besondere Betroffenheit von Fachkräfteengpässen konstatiert (vgl. z. B. Kay/Richter 2010; Kouli 2012; BMWi 2012). Als wesentliche Ursachen gelten spezifische Nachteile, die KMU gegenüber Großunternehmen bei der Deckung des eigenen Nachwuchs- und Fachkräftebedarfs haben (etwa Defizite beim Rekrutierungsmanagement, fehlende strategische Personalarbeit oder eine geringere Arbeitgeberattraktivität). Reize (2011) weist jedoch darauf hin, dass sich angesichts der Heterogenität des KMU-Sektors die Fachkräftesituation von kleineren Unternehmen nicht pauschal beurteilen lässt. Um zu einer entsprechenden Differenzierung zu gelangen, bietet sich daher ein Fokus auf bestimmte KMU-Teilgruppen an, wobei stets die Frage im Raum steht, ob aus Sicht der jeweils zugehörigen Betriebe tatsächlich von einem anhaltenden Ungleichgewicht zwischen Arbeitsangebot und Arbeitsnachfrage auszugehen ist.

Für ein solches Unterfangen stellt die Handwerkswirtschaft erstens bereits aufgrund ihrer kleinbetrieblich geprägten Unternehmensgrößenstruktur ein anschauliches Untersuchungsfeld dar. Nach den Ergebnissen der Handwerkszählung 2008 haben rund $82 \%$ der fast 580.000 Un-

1 Der Autor dankt den beiden anonymen Gutachtern/Gutachterinnen der WSI-Mitteilungen für ihre wertvollen Hinweise und Kommentare. 
ternehmen des zulassungspflichtigen bzw. zulassungsfreien Handwerks (Anlagen A und B1 Handwerksordnung) weniger als zehn Beschäftigte (Tabelle 1). Zweitens liegen inzwischen zwar verschiedene Arbeiten vor, die am Beispiel des Handwerks mögliche Ansatzpunkte und Strategien zur Fachkräftesicherung behandeln (vgl. z. B. Kranzusch et al. 2009; Wolf 2012; Bizer/Thomä 2013). Es fehlt jedoch noch an einer Darlegung der spezifischen Ursachen, die womöglich gerade im Falle des Handwerks für eine ausgeprägte Fachkräfteproblematik sprechen. Ebendies wäre jedoch für die bessere Identifikation von solchen Arbeitsmarktsegmenten relevant, in denen zur Vermeidung von steigenden Fachkräfteengpässen und der damit verbundenen volkswirtschaftlichen Kosten die Notwendigkeit von rechtzeitigen Anpassungsreaktionen seitens der Marktteilnehmer besonders groß ist. Drittens ist freilich in Rechnung zu stellen, dass die mit einer schrumpfenden Bevölkerung einhergehende Senkung des marktseitigen Nachfragevolumens mittel- bis längerfristig auch innerhalb des Handwerks einen dämpfenden Effekt auf den Bedarf an Arbeitskräften haben könnte. Vorliegenden Studien zufolge resultieren aus den demografisch bedingten Veränderungen allerdings gerade in verschiedenen Märkten der Handwerkswirtschaft auch umfangreiche Nachfragepotenziale und Absatzchancen, für deren Realisierung eine ausreichende Fachkräfteversorgung der dortigen Betriebe eine unabdingbare Grundvoraussetzung ist (vgl. Haverkamp 2011; Dürig et al. 2012).

Vor diesem Hintergrund diskutiert der Beitrag die Fachkräftesituation des Handwerks. Die regelmäßige Einnahme einer vergleichenden Perspektive erlaubt hierbei eine Annäherung an die Frage, ob eine bedarfsdeckende Personalversorgung insbesondere für Betriebe der Handwerkswirtschaft eine Herausforderung darstellt. Abschnitt 2 spannt zunächst den Untersuchungsrahmen, indem die aktuelle Lage am Ausbildungsstellen- und Arbeitsmarkt aus der Perspektive des Handwerks beleuchtet wird. Die Abschnitte 3 und 4 widmen sich sodann sowohl auf der Nachwuchs- als auch auf der Fachkräfteebene der Sammlung empirischer Hinweise, welche dazu dienen, die Betroffenheit der Handwerksbetriebe von der Verknappung des Arbeitskräfteangebots möglichst fundiert einzuschätzen. Abschnitt 5 schließt mit einem Fazit und einer Diskussion, in welcher die Implikationen der Untersuchungsergebnisse im Vordergrund stehen.

\section{Eine überproportionale Betroffenheit des Handwerks?}

Nach den Ergebnissen der 12. koordinierten Bevölkerungsvorausberechnung des Statistischen Bundesamtes (Variante 1-W1) wird - bezogen auf das Jahr 2009 - die Altersgruppe der 16- bis unter 20-Jährigen in Deutschland bis 2025 voraussichtlich um rund $22 \%$ zurückgehen. Diese demografische Entwicklung bedeutet, dass die Nachfrage nach Ausbildungs-

\section{TABELLE 1}

Verteilung der Handwerksunternehmen nach Größenklassen, 2008

Angaben in Prozent

\begin{tabular}{l|c|c|c|c|c}
\hline \multicolumn{5}{c}{ Größenklasse (gemessen an der Beschäftigtenzahl) } \\
\cline { 2 - 6 } & unter 5 & $\mathbf{5 - 9}$ & $\mathbf{1 0 - 1 9}$ & $\mathbf{2 0 - 4 9}$ & $\mathbf{5 0}$ und mehr \\
\hline Handwerk insgesamt & 60,6 & 21,0 & 11,0 & 5,3 & 2,1 \\
A-Handwerke & 58,0 & 22,5 & 11,9 & 5,6 & 1,9 \\
B1- Handwerke & 74,5 & 12,9 & 6,3 & 3,6 & 2,8 \\
\hline
\end{tabular}

Quelle: Müller (2012, S. 9) auf Grundlage der Handwerkszählung 2008.

plätzen im dualen System aufgrund rückläufiger Schulabgängerzahlen stark absinkt. Etwa seit dem Jahr 2008 spüren dies die Betriebe in Deutschland in Form von zunehmenden Rekrutierungsengpässen am Ausbildungsstellenmarkt (Autorengruppe Bildungsberichterstattung 2012). Tabelle 2 verdeutlicht die hierdurch veränderte Relation zwischen dem Angebot und der Nachfrage nach Ausbildungsplätzen. Unabhängig von der unterstellten Nachfragedefinition blieben demnach absolut gesehen im Handwerk während des Beobachtungszeitraums überdurchschnittlich viele Ausbildungsstellen unbesetzt: Das Verhältnis zwischen der Zahl der Ausbildungsangebote und der Zahl der Personen, die offiziell als Ausbildungsplatznachfrager ausgewiesen werden, betrug $2009 \mathrm{im}$ handwerklichen Ausbildungsbereich z. B. nach traditioneller Definition noch 101,8 \% (Industrie und Handel: $101,2 \%)$. Bis zum Jahr 2011 hatte sich dieser Indikator bereits auf 104,4 \% erhöht (Industrie und Handel: 103,7 \%).

Dass das Handwerk in besonderem Maße mit Nachwuchsproblemen konfrontiert ist, zeigt sich auch anhand von Ergebnissen des BIBB-Qualifizierungspanels. Hierbei handelt es sich um eine repräsentative Erhebung unter rund 2.000 Betrieben, die vom Bundesinstitut für Berufsbildung (BIBB) in Zusammenarbeit mit TNS Infratest Sozialforschung bisher im Frühjahr 2011 und im Frühjahr 2012 durchgeführt wurde. Auf dieser Grundlage kann untersucht werden, welche Betriebe derzeit eher als andere mit Problemen zu kämpfen haben, ihre Ausbildungsstellen erfolgreich zu besetzen (Tabelle 3). Von den Handwerksbetrieben mit Ausbildungsstellenangeboten für das Ausbildungsjahr 2010/2011 konnten demnach 43,5 \% ihre Ausbildungsplätze teilweise oder vollständig nicht besetzen. Im Ausbildungsbereich „Industrie und Handel“ lag dieser Anteil mit knapp einem Drittel deutlich niedriger. Im folgenden Ausbildungsjahr 2011/2012 berichtete in beiden Fällen bereits ein größerer Anteil der Betriebe von Problemen bei der Besetzung angebotener Ausbildungsplätze.

Eine alternative Rekrutierungsstrategie im Vergleich zu eigenen Ausbildungsanstrengungen (und der anschließenden Übernahme von Ausbildungsabsolventen) ist die Besetzung von offenen Arbeitsstellen mit betriebsfrem- 
TABELLE 2

Angebots-Nachfrage-Relation im dualen Ausbildungssystem*, 2009-2011

Angaben in Prozent

\begin{tabular}{|c|c|c|c|c|c|c|}
\hline \multirow[b]{2}{*}{ Jahr } & \multicolumn{3}{|c|}{$\begin{array}{l}\text { Angebots-Nachfrage-Relation } \\
\text { (traditionelle Definition) }\end{array}$} & \multicolumn{3}{|c|}{$\begin{array}{l}\text { Angebots-Nachfrage-Relation } \\
\text { (erweiterte Definition) })^{1}\end{array}$} \\
\hline & 2009 & 2010 & 2011 & 2009 & 2010 & 2011 \\
\hline Insgesamt & 101,3 & 101,8 & 103,5 & 89,8 & 90,6 & 93,3 \\
\hline \multicolumn{7}{|l|}{ Darunter: } \\
\hline Handwerk & 101,8 & 102,8 & 104,4 & 90,5 & 92,1 & 95,4 \\
\hline Industrie und Handel & 101,2 & 101,6 & 103,7 & 89,3 & 90,0 & 92,9 \\
\hline
\end{tabular}

* differenziert nach Zuständigkeitsbereichen.

1 Nach der erweiterten Definition wird bei der Ermittlung der Ausbildungsplatznachfrage nicht nur die Zahl der neu abgeschlossenen Ausbildungsverträge und die Zahl der unvermittelten/unversorgten Bewerberinnen und Bewerber berücksichtigt, sondern auch die Zahl der noch suchenden Bewerber/-innen mit alternativen Verbleibsmöglichkeiten ( $\mathrm{z}$. B. Beginn eines Praktikums, Teilnahme an Berufsvorbereitungsmaßnahmen). Siehe hierzu Ulrich (2012, S. 52 ff.). Lesebeispiel: Gemäß der traditionellen Definition standen im Jahr 2009 über alle Ausbildungsbereiche hinweg rechnerisch 100 Ausbildungsplatznachfragern 101,3 Ausbildungsplatzangebote gegenüber.

Quelle: Autorengruppe Bildungsberichterstattung (2012, S. 280) auf Grundlage verschiedener Datenquellen.
TABELLE 3

\section{Zur Lage am Ausbildungsstellen- und Arbeitsmarkt*, 2010-2012}

Angaben in Prozent

\begin{tabular}{|c|c|c|c|c|}
\hline \multirow[b]{2}{*}{ Jahr } & \multicolumn{2}{|c|}{$\begin{array}{c}\text { Anteil der Betriebe mit } \\
\text { unbesetzten } \\
\text { Ausbildungsstellen' }\end{array}$} & \multicolumn{2}{|c|}{$\begin{array}{c}\text { Anteil der Betriebe mit } \\
\text { unbesetzten } \\
\text { Arbeitsstellen }^{2}\end{array}$} \\
\hline & $2010 / 2011$ & $2011 / 2012$ & 2010 & 2011 \\
\hline Insgesamt & 34,8 & 37,4 & 32,1 & k.A. \\
\hline \multicolumn{5}{|l|}{ Darunter: } \\
\hline Handwerk & 43,5 & 47,7 & 40,7 & k.A. \\
\hline Industrie und Handel & 31,0 & 35,8 & 28,9 & k.A. \\
\hline
\end{tabular}

* differenziert nach Zuständigkeitsbereichen.

1 Anteil von Betrieben mit unbesetzten Ausbildungsstellen an allen Betrieben mit neuen Ausbildungsstellenangeboten für die Ausbildungsjahre 2010/2011 und 2011/2012.

2 Anteil von Betrieben, die im Kalenderjahr 2010 Arbeitsstellen nicht besetzen konnten, an allen Betrieben, die Arbeitsstellen in diesem Zeitraum angeboten haben.

Quelle: Bundesinstitut für Berufsbildung (2012, S. 207ff.; 2013, S. 234) auf Grundlage des BIBB-Qualifizierungspanels. betrieblichen Berufsausbildung die nötigen Rückschlüsse. Im Rahmen der Erhebung für das Berichtsjahr 2007 wurden knapp 3.000 Ausbildungsbetriebe über alle Branchen und Betriebsgrößenklassen hinweg befragt. Die Ergebnisse machen deutlich, dass es in erster Linie Ausbildungsbetriebe aus dem Handwerk als schwierig erachten, auf dem freien Arbeitsmarkt eine ausreichende Zahl von qualifizierten Arbeitskräften für sich zu gewinnen. Ein überdurchschnittlich großer Anteil der Befragten aus dem Handwerk schätzte z. B. die betriebliche Einsatzfähigkeit der potenziell verfügbaren Arbeitskräfte als schlecht oder sogar sehr schlecht ein (Schönfeld et al. 2010). Diese schwierige Arbeitsmarktposition des Handwerks zeigt sich gut am Beispiel der ersten Erhebungswelle des BIBB-Qualifizierungspanels ( $\mathrm{Ta}$ belle 3). Denn demnach konnten im Jahr 2010 rund $41 \%$ der Handwerksbetriebe kein oder nicht ausreichend qualifiziertes Personal zur Besetzung von offenen Arbeitsstellen finden. In der Industrie und im Handel lag dieser Anteil mit knapp $29 \%$ wesentlich niedriger.

Der Umstand, dass die Nachwuchs- und Fachkräftegewinnung offenbar vor allem für das Handwerk eine Herausforderung darstellt, kann auf das ungünstige Zusammenspiel einer eher schwachen Wettbewerbsposition der Handwerksbetriebe am Ausbildungsstellen- und Arbeitsmarkt auf der einen Seite und eines im Zuge des demografischen Wandels wachsenden personalseitigen Problemdrucks auf der anderen Seite zurückgeführt werden (Mendius 2001, 2003). Insofern lässt sich eine Gemengelage von empirischen Hinweisen anführen, die für eine problematische Fachkräftesituation insbesondere des Handwerks sprechen.

\section{Hinweise auf der Nachwuchsebene}

Zunächst ist die hohe Konzentration des Handwerks auf Ausbildungsaktivitäten zum Zwecke der Personalgewinnung zu nennen. Auf Grundlage der BIBB/BAuA-Erwerbstätigenbefragung 2006 - einer vom BIBB im Zeitraum 2005/2006 gemeinsam mit der Bundesanstalt für Arbeitsschutz und Arbeitsmedizin (BAuA) durchgeführten repräsentativen Erhebung unter erwerbstätigen Personen in Deutschland - beziffert Haverkamp $(2013,2014)$ den Anteil der im Handwerk ausgebildeten und auch später dort verbliebenen Fachkräfte auf rund 70 \%. Diese große Rolle des eigenen Ausbildungsbereichs für die handwerkliche Nachwuchsrekrutierung zeigt sich auch anhand von Tabelle 4. Ermöglicht durch die Umstellung der Handwerkszählung des Statistischen Bundesamts auf eine Auswertung des Unternehmensregisters lässt sich näherungsweise ermitteln, dass der Anteil des Handwerks an allen deutschen Erwerbstätigen mit abgeschlossener Berufsausbildung im mittleren Qualifikationsbereich (d. h. deren höchster Bildungsabschluss der Qualifikationsstufe ISCED 3b \& 4 zugeordnet ist) im Jahr 2010 bei 12,3 \% lag. Laut Berufsbildungsstatis- 
tik der statistischen Ämter des Bundes und der Länder betrug hingegen im selben Jahr der Handwerksanteil am gesamten Auszubildendenbestand fast $29 \%$; ein Umstand, der nicht nur auf eine überdurchschnittlich hohe Ausbildungsquote verweist, sondern auch die handwerkstypische „Ausbildung über eigenen Bedarf" widerspiegelt.

Die umfangreichen Ausbildungsaktivitäten des Handwerks führen seit jeher dazu, dass sich demografische Veränderungen aus Sicht der Handwerksbetriebe zuallererst und besonders deutlich in der Versorgungslage am Ausbildungsstellenmarkt niederschlagen (Mendius 2003). Aktuell ist dies wieder sehr gut zu beobachten. Denn der demografische Wandel hat zwar bisher den gesamten Umfang des verfügbaren Arbeitskräfteangebots in Deutschland noch nicht spürbar reduziert (IAB-Autorengruppe 2012). Wie im vorigen Abschnitt gezeigt, hat er aber bereits merkliche Spuren am Ausbildungsstellenmarkt in Form eines Rückgangs der Nachfrage nach Ausbildungsplätzen hinterlassen. Hiervon sind viele Ausbildungsbetriebe aus dem Handwerk negativ betroffen. Die hohe Ausbildungsquote des Handwerks führt somit dazu, dass die dortigen Betriebe die Folgen des demografischen Wandels auf der Personalseite besonders frühzeitig spüren.

Darüber hinaus haben die Schwierigkeiten auf der handwerklichen Nachwuchsebene eine quantitative und eine qualitative Dimension. Ein überdurchschnittlich großer Anteil der Auszubildenden im Handwerk verfügt nur über eine Hauptschulqualifikation als höchsten allgemeinbildenden Schulabschluss (Tabelle 5). Im Jahr 2011 waren dies 52,0 \%; weitere 3,9\% der handwerklichen Ausbildungsanfängerinnen und -anfänger hatten gar keinen Abschluss. Im Ausbildungsbereich „Industrie und Handel“ beliefen sich diese beiden Anteile im selben Jahr nur auf 24,8 \% bzw. 2,3\%. Entsprechend liegt dort der Anteil der Auszubildenden mit Realschulabschluss oder mit einer Studienberechtigung wesentlich höher als im Handwerk.

Diese Vorbildungsstruktur hat unmittelbare Konsequenzen für die künftige Nachwuchssicherung im Handwerk. Denn die vorliegenden Vorausberechnungen zur weiteren Entwicklung der Schulabgängerzahlen machen deutlich, dass insbesondere die Absolventen allgemeinbildender Schulen, die maximal über einen Hauptschulabschluss verfügen, im Zuge des demografischen Wandels zurückgehen werden. So erwartet die Kultusministerkonferenz (2011) für den Zeitraum 2010 bis 2020 einen Rückgang der Zahl der Hauptschulabsolventen in Höhe von 27 \% (im Vergleich dazu: Absolventen mit Realschulabschluss: $-12 \%$ und Absolventen mit Fachhochschul- und Hochschulreife: -1 \%). Dies hat für das Handwerk zur Folge, dass sich die bisherige Hauptrekrutierungsquelle für die Ausbildung des eigenen Fachkräftenachwuchses in absehbarer Zeit stark verkleinern wird.

In diesem Zusammenhang ist ferner von Bedeutung, dass durch den gesellschaftlichen und technologischen Wandel auch im Handwerk die Höhe und die Dynamik der Qualifikationsanforderungen zugenommen haben. So können etwa Haverkamp et al. (2009) durch eine Gegenüberstellung der

\section{TABELLE 4}

\section{Anteil des Handwerks am gesamtwirtschaftlichen Beschäftigten- und Auszubildendenbestand, 2010}

Angaben in absoluten Zahlen und in Prozent

\begin{tabular}{|c|c|c|c|}
\hline & $\begin{array}{c}\text { Gesamt- } \\
\text { wirtschaft }\end{array}$ & Handwerk & $\begin{array}{c}\text { Anteil des } \\
\text { Handwerks }\end{array}$ \\
\hline $\begin{array}{l}\text { Zahl der Erwerbstätigen mit } \\
\text { abgeschlossener Berufsausbildung } \\
\text { (ISCED } 3 \text { b \& 4) in } 1.000\end{array}$ & 21.660 & $2.669^{1}$ & $12,3 \%$ \\
\hline Zahl der Auszubildenden in 1.000 & 1.508 & 435 & $28,8 \%$ \\
\hline
\end{tabular}

1 Die Zahl der Erwerbstätigen im Handwerk mit abgeschlossener Berufsausbildung im mittleren Qualifikationsbereich wurde auf Grundlage der ZDH-Strukturumfrage 2009 geschätzt. Demnach beläuft sich der Anteil der Gesellen sowie der technischen bzw. kaufmännischen Fachkräfte unter den Erwerbstätigen des Handwerks auf 53,6 \% (Müller 2012, S. 199).

Quelle: Berechnungen des QuBe-Projektes (www.qube-projekt.de, letzter Zugriff: 24.04.2014); Statistisches Bundesamt (2013a, S. 51): Bundesinstitut für Berufsbildung (2013, S. 121) auf Grundlage der Berufsbildungsstatistik.

\section{TABELLE 5}

\section{Verteilung der Auszubildenden mit neu abgeschlossenem Ausbildungs- vertrag nach höchstem allgemeinbildenden Schulabschluss*, 2011 \\ Angaben in Prozent}

\begin{tabular}{l|c|c|c|c}
\hline & $\begin{array}{c}\text { ohne } \\
\text { Hauptschul- } \\
\text { abschluss }\end{array}$ & $\begin{array}{c}\text { Hauptschul- } \\
\text { abschluss }\end{array}$ & $\begin{array}{c}\text { Realschul- } \\
\text { abschluss }\end{array}$ & $\begin{array}{c}\text { Studien- } \\
\text { berechtigung }\end{array}$ \\
\hline $\begin{array}{l}\text { Insgesamt } \\
\begin{array}{l}\text { Darunter: } \\
\text { Handwerk }\end{array}\end{array}$ & $\mathbf{2 , 9}$ & $\mathbf{3 1 , 9}$ & $\mathbf{4 2 , 1}$ & $\mathbf{2 3 , 1}$ \\
\hline \begin{tabular}{l} 
Industrie und Handel \\
\hline
\end{tabular} & 2,9 & 52,0 & 36,1 & 8,1 \\
\hline
\end{tabular}

*differenziert nach Zuständigkeitsbereichen.

Quelle: Bundesinstitut für Berufsbildung (2013, S. 170) auf Grundlage der Berufsbildungsstatistik.
Ergebnisse der BIBB/BAuA-Erwerbstätigenbefragung 2006 mit denen der entsprechenden Vorgängerbefragung aus dem Jahr 1999 aufzeigen, dass sich die handwerklichen Tätigkeitsspektren in den vergangenen Jahren gewandelt haben und infolgedessen die Anforderungen der Betriebe an das Kompetenzniveau von Handwerksbeschäftigen gestiegen sind. Diese gewachsene Bedeutung des Produktionsfaktors ,Wissen“ für den handwerklichen Leistungserstellungsprozess zeigt Dürig (2011) am Beispiel verschiedener Märkte der Handwerkswirtschaft anschaulich auf.

Trotz dieser Entwicklung hat das Handwerk aber in der Vergangenheit den allgemeinen gesellschaftlichen Trend zum Erwerb höherer Bildungsabschlüsse nicht nachvollzogen, was durch den konstant hohen Anteil von Auszubildenden mit geringerer schulischer Vorbildung zum Ausdruck kommt. Eine Ursache hierfür ist sicherlich im vergleichsweise schlechten Image der handwerklichen Ausbildungsberufe zu suchen (Forsa-Institut 2008), wo- 
durch das Handwerk selbst in Zeiten eines Überangebots auf dem Ausbildungsstellenmarkt oftmals nicht in ausreichendem Maße qualifizierte und motivierte Jugendliche für sich gewinnen konnte. Gut erkennbar wird das daraus resultierende Spannungsverhältnis am Beispiel des gerade im Handwerk häufig vorkommenden Problems der Ausbildungsabbrüche. Im Jahr 2011 war der Anteil der vorzeitigen Vertragslösungen an allen begonnenen Ausbildungsverhältnissen im Handwerk unter allen Ausbildungsbereichen mit Abstand am höchsten. Nach Berechnungen des BIBB betrug die entsprechende Lösungsquote bundesweit $31,1 \%$, wohingegen diese im Zuständigkeitsbereich der Industrie- und Handelskammern mit 22,1\% erheblich niedriger ausfiel (Bundesinstitut für Berufsbildung 2013, S. 187). Auch wenn vorzeitig gelöste Verträge keineswegs automatisch mit einem

\section{TABELLE 6}

\section{Durchschnittlicher Bruttomonatsverdienst (inkl. Sonderzahlungen) einer vollzeitbeschäftigten Fachkraft ${ }^{1}$ im Produzierenden Gewerbe und im Dienstleistungsbereich*, 2012}

Angaben in Euro

\begin{tabular}{lc|c}
\hline & Produzierendes Gewerbe & Dienstleistungsbereich \\
\hline Insgesamt & $\mathbf{3 . 2 9 9}$ & $\mathbf{2 . 9 4 7}$ \\
Darunter: & & \\
Handwerk & 2.595 & 2.539 \\
Nicht-Handwerk & 3.584 & 2.974 \\
\hline
\end{tabular}

* differenziert nach Handwerkseigenschaft eines Betriebs.

1 Definition „Fachkraft": Arbeitnehmer mit schwierigen Fachtätigkeiten, für deren Ausübung in der Regel ein abgeschlossene Berufsausbildung, zum Teil verbunden mit Berufserfahrung, erforderlich ist (Leistungsgruppe 3)

\section{TABELLE 7}

\section{Beschäftigungsbereiche der Personen mit Ausbildungsabschluss im westdeutschen Handwerk, 1979-2006}

Angaben in Prozent

\begin{tabular}{lc|c|c|c|c}
\hline Jahr & Handwerk & Industrie & Handel & $\begin{array}{c}\text { Sonstige } \\
\text { Öfentlicher } \\
\text { Dienst }\end{array}$ & $\begin{array}{c}\text { Dienstleistun- } \\
\text { gen, andere }\end{array}$ \\
\hline 2006 & 34,5 & 24,9 & 12,9 & 13,6 & 14,1 \\
1999 & 50,1 & 18,4 & 9,5 & 13,3 & 8,7 \\
1992 & 50,2 & 21,9 & 8,5 & 12,7 & 6,8 \\
1986 & 46,8 & 21,2 & 8,5 & 13,9 & 9,6 \\
1979 & 44,8 & 24,6 & 8,3 & & $22,3^{1}$ \\
\hline
\end{tabular}

1 Gesamtzahl für öffentlicher Dienst und sonstige Dienstleistungen.

Lesebeispiel: Im Jahr 2006 waren von 100 im Handwerk ausgebildeten Fachkräften noch 34,5\% im Handwerk erwerbstätig.

Quelle: Haverkamp et al. (2009, S. 91) auf Grundlage verschiedener BIBB/IAB-Erhebungen und der BIBB/BAuA-Erwerbstätigenbefragung 2006. endgültigen Ausbildungsabbruch gleichzusetzen sind und die Ursachen für Ausbildungsabbrüche vielfältig sein können, dürfte in diesem Zusammenhang dennoch der Umstand von Relevanz sein, dass im dualen System die Vertragslösungsquote bei Auszubildenden ohne und mit Hauptschulabschluss deutlich über dem Durchschnitt liegt (Autorengruppe Bildungsberichterstattung 2012).

\section{Hinweise auf der Fachkräfteebene}

Aus Sicht der Handwerkswirtschaft sind hinsichtlich der zukünftigen Deckung des betrieblichen Personalbedarfs drei weitere Faktoren maßgeblich: Das auf der Fachkräfteebene bestehende Einkommensgefälle zwischen dem handwerklichen und dem nicht-handwerklichen Teil der Wirtschaft, die damit zusammenhängende Abwanderung von im Handwerk ausgebildeten Personen in andere Wirtschaftsbereiche sowie der überproportionale Facharbeiteranteil unter den Handwerksbeschäftigten. Zu Ersterem: Die schwache Arbeitsmarktposition des Handwerks äußert sich seit jeher in Form eines vergleichsweise niedrigen Lohn- und Gehaltsniveaus (Dürig et al. 2004). Im produzierenden Gewerbe, in dem das Handwerk schwerpunktmäßig verankert ist, tritt dieser Umstand besonders deutlich zutage (Tabelle 6). Der durchschnittliche Bruttomonatsverdienst einer vollzeitbeschäftigten Fachkraft belief sich im Jahr 2012 in der Industrie (nicht-handwerkliche Betriebe des produzierenden Gewerbes) auf $3.584 €$; im Handwerk lag dieser hingegen mit $2.595 €$ wesentlich niedriger. Prozentual gesehen betrug der durchschnittliche Verdienstunterschied zwischen Handwerk und Industrie demnach fast $28 \%$.

Neben der Ausbildung über eigenen Bedarf haben in der Vergangenheit vor allem diese geringeren Verdienstmöglichkeiten die "traditionelle“ Abwanderung der im Handwerk ausgebildeten Personen in andere Wirtschaftsbereiche begünstigt (Mendius 2003). Der personalseitige Problemdruck, welcher hieraus in den Handwerksbetrieben erwächst, dürfte sich jedoch gerade in jüngerer Zeit vergrößert haben. Denn nach den Ergebnissen von Haverkamp et al. (2009) waren $2006 \mathrm{im}$ westdeutschen Handwerk nur noch rund $35 \%$ derjenigen Personen, die eine handwerkliche Erstausbildung absolviert hatten, auch immer noch im Handwerk erwerbstätig (Tabelle 7). Im Jahr 1999 lag dieser Anteil noch bei $50 \%$. Im Gegenzug hat sich zwischen 1999 und 2006 entsprechend der Anteil an Personen erhöht, die ursprünglich im westdeutschen Handwerk ihre Berufsausbildung absolviert hatten und nun in der Industrie oder anderen Wirtschaftsbereichen beschäftigt sind. Einerseits bedeutet diese Entwicklung, dass das Handwerk offenbar auch weiterhin als wichtiger Arbeitskräftelieferant für andere Wirtschaftsbereiche wie die Industrie oder den Handel fungiert. Andererseits macht der gesunkene Verbleibsanteil deutlich, dass sich die Abwanderung aus dem Handwerk 
im Vergleich zur Vergangenheit erhöht hat. Interessant erscheint in diesem Zusammenhang der Befund von Scholz (2013, S. 202ff.), wonach etwa seit Ende der 1990er Jahre die im Handwerk gezahlten Löhne und Gehälter zunehmend stärker hinter denen im nicht-handwerklichen Teil der Wirtschaft zurückgefallen sind.

Im Falle des ostdeutschen Handwerks ist für den gleichen Zeitraum (d. h. zwischen 1999 und 2006) ein ähnlich starker Rückgang des relativen Verbleibs von selbst ausgebildeten Fachkräften zu verzeichnen. Aus qualitativer Sicht kommt hinzu, dass es sich in beiden Teilen der Bundesrepublik bei den abwandernden Personen oftmals um Jüngere gehandelt hat. Daneben fällt auf, dass insbesondere Ausbildungsabsolventen mit Fachhochschul- oder Hochschulreife aus dem Handwerk abgewandert sind. Zumindest im westdeutschen Handwerk hat sich im Betrachtungszeitraum zwar auf der anderen Seite auch der Anteil derjenigen Personen erhöht, welche aus anderen Wirtschaftsbereichen in das Handwerk zuwandern. Wie Haverkamp (2013; 2014) aufzeigt, handelt es sich hierbei jedoch vergleichsweise häufig um Fachkräfte mit kaufmännischer Qualifikation; also um Personen aus solchen Berufsgruppen, die in der Handwerkswirtschaft einerseits wenig ausgebildet werden und bei denen andererseits weder derzeit noch in Zukunft ausgeprägte Rekrutierungsengpässe auftreten dürften (BMWi 2012; IAB-Autorengruppe 2012). Nicht nur aufgrund ihrer vergleichsweise geringen quantitativen Bedeutung dürfte daher im Handwerk die sektorübergreifende Zuwanderung von extern ausgebildeten Personen die mit den fortwährenden Abwanderungstendenzen verbundene Gefährdung der eigenen Fachkräftesicherung kaum kompensieren können.

Einen dritten Hinweis liefert die Qualifikationsstruktur der Handwerksbeschäftigten. Nach den Ergebnissen einer Strukturumfrage, die der Zentralverband des Deutschen Handwerks (ZDH) im Jahr 2009 unter 12.000 Betrieben durchgeführt hat, beläuft sich der Anteil der Gesellen bzw. technischen Fachkräfte unter den Handwerksbeschäftigten auf knapp 45 \% sowie der Anteil der kaufmännischen Fachkräfte auf $9 \%$. An- und ungelernte Arbeitskräfte haben im Handwerk demgegenüber mit etwa $15 \%$ ein weitaus geringeres Gewicht. Dieser Anteil würde sogar noch einmal deutlich kleiner ausfallen, wenn der beschäftigungsstarke Handwerkszweig der Gebäudereiniger unberücksichtigt bliebe. Hier ist der Anteil der An- und Ungelernten unter den Beschäftigten besonders hoch, im Jahr 2009 lag er bspw. bei rund $78 \%$ (Müller 2012).

Auch wenn in diesem Falle keine aktuellen Vergleichsdaten für die Gesamtwirtschaft vorliegen, spricht somit vieles dafür, dass Arbeitskräfte der mittleren Qualifikationsstufe für die Personalversorgung des Handwerks von besonderer Bedeutung sind. Diese Vermutung wird durch die Untersuchung von Haverkamp et al. (2009) gestützt. Auf Grundlage der BIBB/IAB-Erhebung 1998/1999 - eine Repräsentativbefragung von Erwerbstätigen, die gemeinsam vom BIBB und vom Institut für Arbeitsmarkt- und Berufsforschung (IAB) durchgeführt wurde - zeigen die
Autoren, dass der Anteil der Erwerbstätigen ohne abgeschlossene Berufsausbildung im Handwerk niedriger ausfällt als z. B. in der Industrie (10,9 \% vs. 15,5\%), wohingegen unter den Handwerksbeschäftigten der Anteil an Personen, die unterhalb der Hochschulebene ihre Berufsausbildung abgeschlossen haben, entsprechend größer ist (70,4 \% vs. 59,9 \%). Insofern ist anzunehmen, dass gerade mit Blick auf das Handwerk die Gefahr einer massiven Verschärfung der derzeitigen Fachkräftesituation besteht. Denn einer vorliegenden Arbeitsmarktprojektion zufolge kann unter Status-quo-Bedingungen (d. h. bei unverändertem Verhalten der Marktteilnehmer) auf absehbare Zeit in Deutschland primär der Bedarf an Arbeitskräften mit abgeschlossener Berufsausbildung nicht mehr gedeckt werden. Auf der mittleren Qualifikationsstufe mit ihrem Schwerpunkt auf Erwerbstätigen mit dualem Ausbildungsabschluss würde sich in der Folge ein Nachfrageüberhang einstellen (Abbildung 1; Maier et al. 2014).

Aus gesamtwirtschaftlicher Sicht ist zwar zu betonen, dass ein dauerhaftes Ungleichgewicht in diesem Ausmaß kaum zu erwarten ist. Denn eine demografisch bedingte Schrumpfung des verfügbaren Arbeitskräfteangebots wird zu komplexen Anpassungsreaktionen auf den Güter-, Kapital- und Arbeitsmärkten führen, wodurch letztlich auch die Arbeitskräftenachfrage sinken dürfte (IAB-Autorengruppe 2012). Angesichts der begrenzten Möglichkeiten kleiner Handwerksbetriebe, im Wettbewerb um qualifizierte Fachkräfte mittels Lohnsteigerungen zu punkten, dürften sich vor diesem Hintergrund nichtsdestotrotz vor allem die Stellenbesetzungsengpässe in der Handwerkswirtschaft zunächst noch weiter verschärfen.

ABB. 1

Erwerbstätige und Erwerbspersonen nach Qualifikationsniveau, 2005-2030

Angaben in Millionen Personen

— Erwerbspersonen (Arbeitskräfteangebot) — Erwerbstätige (Arbeitskräftebedarf)

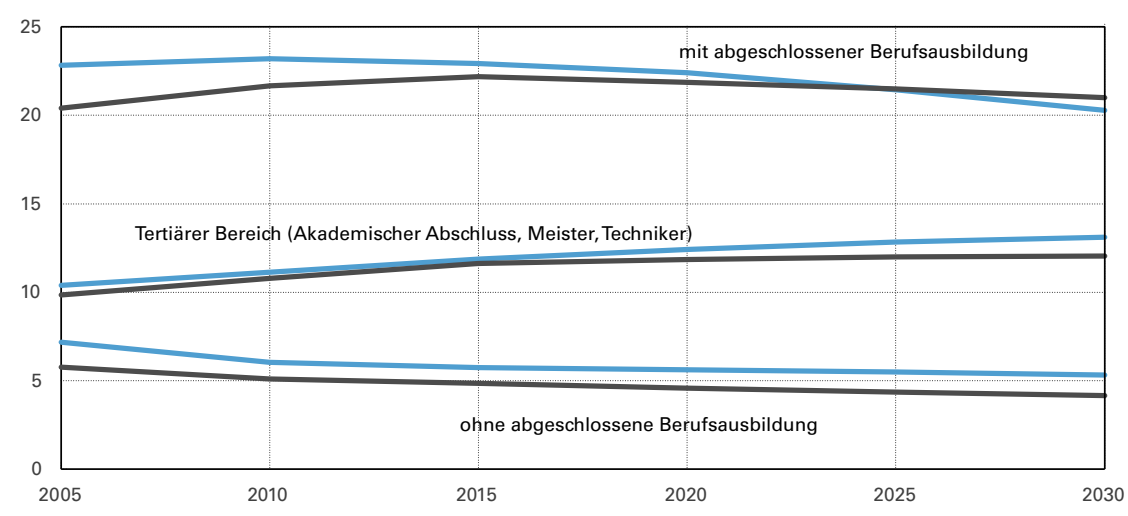

Quelle: QuBE-Projekt, www.qube-projekt.de. 


\section{Fazit und abschließende Diskussion}

Gegenstand des vorliegenden Beitrags ist die Fachkräftesituation in der kleinbetrieblich geprägten Handwerkswirtschaft. Im Ergebnis zeigt sich, dass Handwerksbetriebe aktuell am Ausbildungsstellen- und Arbeitsmarkt vergleichsweise häufig mit Engpässen bei der Besetzung offener Stellen konfrontiert sind, welche sich aller Voraussicht nach in Zukunft noch vergrößern dürften. Hinsichtlich der Möglichkeiten zur Deckung des Nachwuchs- und Fachkräftebedarfs sind dabei aus Sicht des Handwerks letztlich zwei miteinander eng verknüpfte - Faktoren maßgeblich: Erstens ist die Arbeitsmarktposition der Handwerksbetriebe seit jeher relativ schwach: angesichts geringerer Verdienstmöglichkeiten und (tatsächlich oder vermeintlich) unattraktiverer Arbeitsbedingungen als in anderen Wirtschaftsbereichen. Die Folge sind Rekrutierungs- und Personalbindungsprobleme, welche Handwerksbetriebe in der Vergangenheit noch durch bedarfsübersteigende Ausbildungsanstrengungen weitgehend kompensieren konnten. Allerdings hat sich die Tendenz, dass im Handwerk ausgebildete Fachkräfte in andere Wirtschaftsbereiche abwandern, offenbar gerade in jüngerer Zeit verstärkt; eine Entwicklung, die unter anderem mit dem unterdurchschnittlichen Lohn- und Gehaltsniveau im Handwerk zusammenhängen könnte.

Zweitens wird das aus einer schwachen Arbeitsmarktposition resultierende Personalproblem des Handwerks durch den demografischen Wandel massiv verstärkt. Aufgrund ihrer hohen Ausbildungsquote spüren viele Handwerksbetriebe bereits heute den demografisch bedingten Rückgang der Nachfrage nach Ausbildungsplätzen. Hinzu kommt, dass in den kommenden Jahren in erster Linie die Zahl der Schulabgänger und -abgängerinnen, die maximal über einen Hauptschulabschluss verfügen, zurückgehen wird. Dies hat für das Handwerk zur Folge, dass sich die bisherige Hauptrekrutierungsquelle für die Ausbildung des eigenen Fachkräftenachwuchses in absehbarer Zeit deutlich verkleinert. Zudem wächst der Druck angesichts der Tatsache, dass primär im Falle von Erwerbspersonen mit dualer oder vollzeitschulischer Berufsausbildung die potenzielle Gefahr eines übergreifenden Fachkräftemangels besteht. Da Arbeitskräfte des mittleren Qualifikationsbereichs unter den Handwerksbeschäftigten mit Abstand dominieren und angesichts der schwachen Arbeitsmarktposition der meisten Handwerksbetriebe, wird das Handwerk unter Status-quoBedingungen (d. h. im Falle des Ausbleibens von rechtzeitigen Anpassungsreaktionen) von dieser Entwicklung überproportional betroffen sein.

Vor diesem Hintergrund wird deutlich, dass Handwerksbetriebe vor erheblichen Herausforderungen stehen. Um Engpässe auf der Nachwuchsebene abzufedern, gilt es daher auf der einen Seite, noch stärker als bisher auch Jugendlichen mit schlechten Startchancen eine berufliche Perspektive im Handwerk zu eröffnen. Denn für die Zukunft ist weiterhin von einer großen Zahl an jungen Menschen auszugehen, die zwar ein grundsätzliches Interesse an einer dualen Berufsausbildung haben, deren Integration in das duale Ausbildungssystem sich bisher aber schwierig gestaltet und die stattdessen z. B. im sogenannten „Übergangssystem“ landen oder ihr Erwerbsleben ohne abgeschlossene Ausbildung beginnen (Maier et al. 2011). Das Handwerk wird auf diesem Wege auch in Zukunft eine volkswirtschaftlich wichtige Funktion erfüllen.

Auf der anderen Seite macht es der Wandel der handwerklichen Tätigkeitsspektren erforderlich, gleichzeitig mehr motivierte und leistungsstarke Schulabgänger und -abgängerinnen für eine Ausbildung im Handwerk zu gewinnen. Eine Grundvoraussetzung hierfür ist, dass das Handwerk in der Lage ist, seine defizitäre Stellung am Ausbildungsstellenmarkt zu verbessern. Ein gezielter Ansatzpunkt bildet in diesem Zusammenhang die kontinuierliche Sicherstellung einer qualitätsorientierten Ausbildung in Handwerksbetrieben. Die hierdurch erreichbare Erhöhung der individuellen Zufriedenheit von Auszubildenden dürfte letztlich auch zur Senkung der hohen Abbruchquote im handwerklichen Ausbildungsbereich beitragen. Daneben müssen handwerkliche Karrierewege aus Sicht von leistungsstarken jungen Nachwuchskräften attraktiv genug sein. In diesem Zusammenhang ist etwa an eine Fortführung der bisherigen Anstrengungen zu denken, die auf eine höhere Gleichwertigkeit und Durchlässigkeit zwischen dem Berufsbildungs- und dem Hochschulbereich abzielen.

Auf der Fachkräfteebene wird die Steigerung der Arbeitgeberattraktivität von Handwerksbetrieben immer dringlicher. Neben der allgemeinen Notwendigkeit zur besseren Ausschöpfung vorhandener Personalreserven (z. B. hinsichtlich der Erwerbsbeteiligung von Frauen und Älteren) ist aus Sicht des Handwerks in diesem Zusammenhang entscheidend, die Verbleibsbereitschaft von selbst ausgebildeten Fachkräften zu erhöhen. Handwerksbetriebe, die früher noch über den eigenen Bedarf ausbilden konnten, sollten daher stärker dafür sensibilisiert werden, dass frühzeitig gemachte Übernahmeangebote großen Einfluss auf die Entscheidung von jungen Ausbildungsabsolventen haben, im Handwerk zu verbleiben oder in andere Wirtschaftsbereiche abzuwandern (siehe hierzu Wolf 2012). Wagner und Wolf (2013) arbeiten diesbezüglich zudem heraus, dass Handwerksbetriebe einer Abwanderung von Ausbildungsabsolventen durch die stärkere Herausstellung von nicht-monetären Anreizen entgegenwirken können (z. B. mittels Faktoren wie Freude an der Arbeit, regionale Nähe, das Betriebsklima oder Arbeitsplatzsicherheit). Handwerksorganisationen wie Kammern und Verbände können die Betriebe hierbei sehr gut unterstützen, beispielsweise durch eine bedarfsgerechte Anpassung von Ausbildungsinhalten oder Imagemaßnahmen.

Wenn sich die derzeitigen Fachkräfteengpässe in der Handwerkswirtschaft in Zukunft jedoch tatsächlich weiter verschärfen sollten, wird sich das Handwerk spätestens dann auch kritisch mit der Frage auseinandersetzen müssen, in- 
wieweit Lohnsteigerungen möglich sind, um im Wettbewerb um qualifizierte Fachkräfte zu bestehen. Dabei muss klar sein, dass die Antwort hierauf angesichts der großen Heterogenität der Handwerkswirtschaft durchaus unterschiedlich ausfallen kann (Maier et al. 2014, S. 13). Mit Blick auf die Arbeitskräfteknappheit in solchen Handwerksberufen, die durch besonders hohe Anforderungen an personengebundene Kreativität und Spezifität von Fachwissen geprägt sind (z. B. Elektrotechniker, Feinwerkmechaniker oder Tischler), erscheint die Erreichung eines wettbewerbsfähigen Lohnniveaus zumindest nicht von vorneherein ausgeschlossen. Entsprechende Überlegungen sind hier also durchaus gerechtfertigt. In handwerklichen Berufsfeldern, für die eine stark routinegeprägte Leistungserstellung charakteristisch ist und in denen die Entlohnung bereits heute vergleichsweise niedrig ist (z. B. im Lebensmittelgewerbe), könnte ein zu starker Fokus auf die Lohnfrage hingegen dazu führen, dass in den zugehörigen Betrieben mittels technischer und organisatorischer Innovationen zunehmend Tätigkeiten auf An- und Ungelernte übertragen werden. Bestehende Fachkräfteengpässe könnten sich dann in der Folge einer zunehmenden Dequalifizierung des Mitarbeiterstamms von selbst „auflösen“. Um diesem Szenario vorzubeugen, steht das Handwerk daher vor der großen Herausforderung, die Attraktivität von solchen Berufen primär mittels alternativer (d. h. nicht-monetärer) Strategien zu steigern.

Abschließend ist auf die Notwendigkeit von weiterführenden Forschungsarbeiten zu verweisen. Ein Beispiel ist der Umstand, dass aufgrund fehlender empirischer Daten im vorliegenden Beitrag nicht diskutiert werden konnte, welche etwaige Bedeutung die Zuwanderung aus dem Ausland für die Fachkräfteversorgung des Handwerks hat. Angesichts der im Zuge der Eurokrise deutlich angestiegenen Nettozuwanderung nach Deutschland und aufgrund der kürzlich verbesserten Möglichkeit zur Anerkennung von ausländischen Berufsabschlüssen wäre es nicht nur interessant, sondern auch an der Zeit, dieser Frage empirisch nachzugehen. Ein anderes Beispiel sind die noch weitgehend unklaren Konsequenzen, welche sich aus einer Lockerung der rechtlichen Zugangsvoraussetzung zur Ausübung eines Handwerksberufes ergeben. Die entsprechenden Folgen der Novellierung der Handwerksordnung im Jahr 2004 sind z. B. im Hinblick auf das Identitätsbewusstsein im Handwerk untersucht worden (vgl. Müller 2011). Nicht berücksichtigt wurde bisher, dass Zugangsregulierungen einen positiven Einfluss auf die Höhe des Arbeitsentgelts in den betreffenden Berufen haben (vgl. Kleiner 2000; Kleiner/Krueger 2013). Insofern könnten zukünftige Forschungsarbeiten am Beispiel der Handwerksnovelle 2004 analysieren, ob die anschließende Lohnentwicklung in den zulassungsfrei gestellten Gewerken eine eventuelle Steigerung der Arbeitgeberattraktivität von Handwerksbetrieben behindert hat.

\section{LITERATUR}

Autorengruppe Bildungsberichterstattung (2012): Bildung in Deutschland 2012. Ein indikatorengestützter Bericht mit einer Analyse zur kulturellen Bildung im Lebenslauf, Bielefeld

Beicht, U./Walden, G./Herget, H. (2004): Kosten und Nutzen der betrieblichen Berufsausbildung in Deutschland, Bielefeld

Bizer, K./Thomä, J. (Hrsg.) (2013): Fachkräftesicherung im Handwerk, Göttinger Handwerkswirtschaftliche Studien (90), Duderstadt

Bundesinstitut für Berufsbildung (2012): Datenreport zum Berufsbildungsbericht 2012. Informationen und Analysen zur Entwicklung der beruflichen Bildung, Bonn

Bundesinstitut für Berufsbildung (2013): Datenreport zum Berufsbildungsbericht 2013. Informationen und Analysen zur Entwicklung der beruflichen Bildung, Bonn
Bundesministerium für Wirtschaft und Technologie (BMWi) (2012): Fachkräfte sichern - Engpassanalyse, Berlin

Dürig, W. (2011): Dynamik der Märkte. Was bedeutet das für die Identität des Handwerks?, in: Cramer, G./Müller, K. (Hrsg.): Quo vadis Handwerk? Identität des Handwerks im Wandel, Göttinger Handwerkswirtschaftsliche Studien (82), Duderstadt, S. 45-102

Dürig, W./Lagemann, B./Rothgang, M./Trettin, L./Welter, F. (2004): Determinanten des Strukturwandels im deutschen Handwerk. Band I - Schlussbericht, Rheinisch-Westfälisches Institut für Wirtschaftsforschung (RWI), Studie im Auftrag des Bundesministeriums für Wirtschaft und Arbeit, Essen

Dürig, W./Eckl, V./Grunert, P./Lageman, B./Peistrup, M./Trettin, L. (2012): Entwicklung der Märkte des Handwerks und betriebliche Anpassungserfordernisse -Teil I: Analyse. Rheinisch-Westfälisches Institut für Wirtschaftsforschung (RWI), Endbericht zum Forschungsvorhaben (37/09) des Bundesministeriums für Wirtschaft und Technologie, Essen

Forsa-Institut (2008): Das Bild des deutschen Handwerks bei den Bürgern. Ergebnisse einer Forsa-Umfrage, Gesellschaft für Sozialforschung und statistische Analyse mbH, Berlin.

Haverkamp, K. (2011): Nachfragepotenziale und Absatzchancen des Handwerks im demografischen Wandel, Göttinger Handwerkswirtschaftliche Arbeitshefte (69), Duderstadt

Haverkamp, K. (2013): Sektorübergeifende Mobilität: Eine Option der Fachkräftesicherung für das Handwerk?, in: Bizer, K./Thomä, J. (Hrsg.): Fachkräftesicherung im Handwerk, Göttinger Handwerkswirtschaftliche Studien (90), Duderstadt, S. 107-126

Haverkamp, K. (2014): Intern und extern rekrutierte Fachkräfte im Handwerk Verwertung beruflicher Qualifikationen und Einkommen im Vergleich, in: BWP: Demografie und Region 2/2014, S. 51-55

Haverkamp, K./Sölter, A./Kröger, J. (2009): Humankapitalbildung und Beschäftigungsperspektiven im Handwerk, Göttinger Handwerkswirtschaftliche Studien (79), Duderstadt

IAB-Autorengruppe (2012): Fachkräftebedarf: Analysen und Handlungsstrategien, in: Brücker, H./Klinger, S./Möller, J./Walwei, U. (Hrsg.): Handbuch Arbeitsmarkt 2013. Analysen, Daten, Fakten: Institut für Arbeitsmarkt- und Berufsforschung, Bielefeld, S. 205-290

Kay, R./Richter, M. (2010): Fachkräftemangel im Mittelstand: Was getan werden muss. Expertise im Auftrag des Arbeitskreises Mittelstand der Friedrich-EbertStiftung, WISO Diskurs, Ausgabe März, Bonn

Kleiner, M. M. (2000): Occupational licensing, in: Journal of Economic Perspectives 14 (4), S. 189-202

Kleiner, M. M./Krueger, A. B. (2013): Analyzing the extent and influence of occupational licensing on the labor market, in: Journal of Labor Economics 31 (2) S. 173-202

Kouli, E. (2012): Fachkräftesicherung in kleinen und mittleren Unternehmen, in: Zehrfeld, W. (Hrsg.): Fachkräftesicherung. Situation - Handlungsfelder Lösungen, Frankfurt a. M., S. 55-66

Kranzusch, P./Suprinovič, O./Kay, R. (2009): Absatz- und Personalpolitik des Handwerks im Zeichen des demografischen Wandels, IfM-Materialien (188), Bonn

Kultusministerkonferenz (2011): Vorausberechnung der Schüler- und Absolventenzahlen 2010 bis 2025. Statistische Veröffentlichungen der Kultusministerkonferenz (192), August, Berlin

Maier, T./Troltsch, K./Walden, G. (2011): Längerfristige Entwicklung der dualen Ausbildung. Eine Projektion der neu abgeschlossenen Ausbildungsverträge bis zum Jahr 2020, in: Berufsbildung in Wissenschaft und Praxis 3/2011, S. 6-8 Maier, T./Zika, G./Wolter, M. I./Kalinowski, M./Helmrich, R. (2014): Engpässe im mittleren Qualifikationsbereich trotz erhöhter Zuwanderung, BIBB-Report 23/14, Bonn

Mendius, H. G. (2001): Das Handwerk und seine Beschäftigten - Verlierer des demographischen Umbruchs?, in: Aus Politik und Zeitgeschichte (3-4), S. $23-30$

Mendius, H. G. (2003): Fachkräfteversorgung im Handwerk bei alternder Bevölkerung, in: Seminar für Handwerkswesen (Hrsg.): Fachkräftesicherung im Handwerk vor dem Hintergrund struktureller Wandlungen der Arbeitsmärkte, Duderstadt, S. 119-175

Müller, K. (2011): Rückgang des Identitätsbewusstseins im Handwerk: Konsequenzen aus einer Lockerung der Zugangsvoraussetzungen, in: Cramer, G./ Müller, K. (Hrsg.): Quo vadis Handwerk? Identität des Handwerks im Wandel, Göttinger Handwerkswirtschaftliche Studien (82), Duderstadt, S. 103-140 Müller, K. (2012): Analyse der Handwerkszählung 2008, Göttinger Handwerkswirtschaftliche Studien (85), Duderstadt 
Reize, F. (2011): Fachkräftemangel im Mittelstand: Generelles oder partielles Problem?, KFW-Research, Akzente 41/2011

Scholz, J. (2013): Krise des korporatistischen Arrangements und gewerkschaftliche Revitalisierungsansätze im Handwerk, in: Schmalz, S./Dörre, K. (Hrsg.):

Comeback der Gewerkschaften? Machtressourcen, innovative Praktiken, internationale Perspektiven, Frankfurt a. M., S. 199-212

Schönfeld, G./Wenzelmann, F./Dionisius, R./Pfeifer, H./Walden, G. (2010):

Kosten und Nutzen der dualen Ausbildung aus Sicht der Betriebe. Ergebnisse der vierten BIBB-Kosten-Nutzen-Erhebung, Bonn

Statistisches Bundesamt (2013a): Unternehmen, tätige Personen und Umsatz im Handwerk. Berichtsjahr 2010. Fachserie 4 Reihe 7.2, Wiesbaden

Statistisches Bundesamt (2013b): Verdienste und Arbeitskosten: Arbeitnehmerverdienste. Jahr 2012. Fachserie 16 Reihe 2.3, Wiesbaden

Ulrich, J. G. (2012): Indikatoren zu den Verhältnissen auf dem Ausbildungsstellenmarkt, in: Dionisius, R./Lissek, N./Schier, F. (Hrsg.): Beteiligung an beruflicher Bildung. Indikatoren und Quoten im Überblick, Wissenschaftliche Diskussionspapiere des Bundesinstituts für Berufsbildung (133), Bonn, S. 48-65
Wagner, R./Wolf, M. (2013): What drives the intention of Bavarian crafts apprentices to change employer or occupation? An empirical study in the crafts sector, in: Journal for Labour Market Research 46 (1), S. 43-60

Wolf, M. (2012): Fachkräftesicherung in Handwerksbetrieben. Empirische Analy se der Entscheidung junger Fachkräfte für Handwerk oder Industrie, München

\section{AUTOR}

JÖRG THOMÄ, Dr., ist Wissenschaftlicher Mitarbeiter am Volkswirtschaftlichen Institut für Mittelstand und Handwerk (ifh) an der Universität Göttingen. Arbeitsschwerpunkte: Handwerks- und KMU-Forschung

@joerg.thomae@wiwi.uni-goettingen.de 